\title{
L'éducation comme entreprise
}

Légitimations marchande, civique et culturelle de l'école bilingue au Burkina Faso

\section{Géraldine André}

\section{OpenEdition}

\section{Journals}

Édition électronique

URL : http://journals.openedition.org/apad/3993

DOI : 10.4000/apad.3993

ISSN : 1950-6929

Éditeur

LIT Verlag

\section{Édition imprimée}

Date de publication : 15 juin 2009

Pagination : $39-54$

Référence électronique

Géraldine André, "L'éducation comme entreprise », Bulletin de l'APAD [En ligne], 29-30 | 2009, mis en ligne le 16 juin 2010, consulté le 07 septembre 2020. URL : http://journals.openedition.org/apad/3993 ; DOI : https://doi.org/10.4000/apad.3993

Ce document a été généré automatiquement le 7 septembre 2020

Bulletin de l'APAD 


\title{
L'éducation comme entreprise
}

\author{
Légitimations marchande, civique et culturelle de l'école bilingue au
} Burkina Faso

\section{Géraldine André}

1 À travers l'étude de la trajectoire "exemplaire ». d'un entrepreneur et de son initiative dans l'enseignement de base au Burkina Faso, ce texte vise à apporter des éléments de réponse à certaines questions soulevées par l'entrepreunariat africain. La multiplication des écoles bilingues dans le champ scolaire invite à s'interroger sur le type de compétences que mobilisent leurs initiateurs dans un contexte de forte prégnance de l'aide décentralisée. Cette étude de cas consacrée à la genèse et l'expansion de l'éducation bilingue ${ }^{2}$ permet, en outre, d'analyser l'évolution du système éducatif burkinabè caractérisé par une forte privatisation.

\section{Le progressif désengagement de l'État}

\section{Du PAS à la privatisation du système éducatif}

2 Au Burkina Faso, à l'instar de ce qui se produit dans un grand nombre d'autres pays de la sous région, l'éducation est un champ largement ouvert aux initiatives privées. Depuis une dizaine d'années, l'enseignement de base diversifie son offre éducative. A côté de l'offre de l'enseignement public, les innovations pédagogiques sous la coupe de partenaires techniques et financiers prolifèrent (Pilon, 2002). De plus, depuis les années 1990, on observe « une croissance régulière et plus marquée du secteur privé, dont la part atteint $12 \%$ en 2001, représentant près de 110000 élèves » (Pilon, 2002: 8) et $13 \%$ en 2004, atteignant un effectif de plus de 131939 élèves (MEBA : 2004). Selon MarieFrance Lange, «l'évolution de l'offre et de la demande est déterminée par l'influence respective des différents acteurs de l'éducation ». Jusqu'à la fin des années 1970, dans la plupart des pays africains, l'État jouait un rôle central (Lange, 2003 : 147).

La remise en question de cette situation intervient au Burkina Faso en 1987 avec l'avènement $d u$ Front Populaire qui engage l'économie du pays dans un programme 
d'ajustement structurel consacrant le «triomphe de l'idéologie libérale », « le retrait de l'État », "la généralisation de l'économie marchande» (Lange, 2003: 147) et la domination de l'orthodoxie économiste des institutions de Bretton Woods sur l'éducation. Dans l'application de l'économie à l'éducation, les politiques et les choix éducatifs sont évalués en fonction de leur rentabilité mesurée selon le rapport entre les coûts et le taux de scolarisation (Bianchini, 2004 : 179-184). La structuration du champ de l'enseignement de base par ce référentiel est liée en grande partie au contexte de globalisation néo-libérale qui impose face à la souveraineté de l'État d'autres acteurs nationaux et internationaux (Tidjani Alou, 2001). Cette évolution transparaît dans le Plan décennal de développement de l'éducation de base 2000-2009adopté par décret en juillet 1999 et rendu effectif dès la rentrée scolaire 2001-2002. L'initiative privée et la prise en main de l'éducation par les populations locales y sont vivement encouragées; les processus de décentralisation y sont affirmés ${ }^{3}$. L'État y présente en fait son propredésengagement et délègue une grande partie de ses responsabilités aux populations locales et au tissu associatif soutenu par des partenaires techniques et financiers. C'est ce contexte spécifique qui va favoriser la prolifération de l'éducation bilingue.

\section{La reconversion des fonctionnaires}

4 L'éducation bilingue est née à Nomgana, un village situé à une vingtaine de kilomètres de Ouagadougou dans le département de Loumbila. Originaire de Nomgana, M. Pascal, le promoteur de cette entreprise d'éducation bilingue, a bénéficié d'une longue scolarité jusqu'à l'université de Ouagadougou où il a étudié la linguistique. Après l'obtention de son certificat de troisième cycle, il trouve un poste dans une institution publique, l'INA ${ }^{4}$, l'Institut National de l'alphabétisation, qui, dans les années 1980, s'occupe notamment des programmes d'alphabétisation des adultes en langues locales ${ }^{5}$. En 1986, des personnes de son village viennent solliciter son aide "pour amener le développement $\aleph^{6}$ par le biais de la construction d'un centre d'alphabétisation. De par son réseau de relations acquises au sein de l'INA autour de laquelle gravitent une série d'ONG, il répond à ces requêtes par la création d'un centre d'alphabétisation. Ce centre permet à un certain nombre de villageois d'apprendre à lire et à écrire dans leur langue, le moore. Cette initiative suscite des jalousies de villageois voisins qui conduisent ceux de Nomgana à aller voir une nouvelle fois leur " fils " ${ }^{7}$. Fortement sollicité par les habitants des villages alentours, M. Pascal devient progressivement un « courtier en développement» (Bierschenk, Chauveau et Olivier de Sardan, 2000).

5 Skinner note que, déjà dans les années 1960, de nombreux fonctionnaires ouagalais nés en milieu rural cherchaient pour des raisons affectives à investir leur argent dans leur terroir dont ils continuaient d'apprécier la vie «traditionnelle». Cet investissement dans leur village leur procure également une sécurité économique présente et future (Skinner, 1974). Dans le cas de M. Pascal, «le détour par le terroir» (Banegas et Warnier, 2001) peut être interprété comme une forme d'attachement à des principes de solidarité. Mais aussi, les « cadres originaires » sont souvent sollicités par les ruraux de leur village pour la position administrative qu'ils occupent; il leur est difficile de se soustraire à ces «exigences collectives " (Bierschenk, Chauveau et Olivier de Sardan, 2000). Par ailleurs, répondre aux requêtes des siens peut déboucher sur une reconversion professionnelle rentable à l'instar de M. Pascal. Sa trajectoire informe sur 
les occasions entrepreunariales offertes par le contexte actuel dans des domaines particulièrement investis par les structures de développement tels que l'éducation et d'une manière plus générale, la formation.

6 Ce type de reconversion du fonctionnariat est loin d'être inédit au Burkina Faso. Comme l'explique Pascal Labazée, pour pallier son manque d'entrepreneurs, la Première République propulse en 1960 quelques uns de ses cadres comme hommes d'affaires (Labazée, 1988). Dans les années 1970, ce genre de reconversions s'intensifie. Cette nouvelle catégorie de fonctionnaires se distingue nettement, à l'époque, des grands commerçants parce qu'ils effectuent leurs activités moins en fonction « du rendement du capital investi » et de « leurs compétences de gestionnaires » que selon « les compétences techniques » acquises dans le cadre de la fonction publique (Labazée, 1988). L'auteur donne l'exemple d'anciens professeurs et responsables d'établissements scolaires qui s'orientent vers le marché de l'enseignement pour lancer leur entreprise. Non seulement leur expérience leur offre de nombreuses compétences pour concrétiser leur projet, mais aussi elle est un gage de sérieux qui les dote d'un capital symbolique important pour la réussite de leur entreprise. Le capital social constitué au cours de leur expérience dans les ministères et les autres institutions publiques et parapubliques est également une condition " pour débloquer des fonds » et « emporter l'adhésion des décideurs politiques» (Labazée, 1988). Ces figures managériales issues du fonctionnariat élaborent ainsi leur succès sur la conjonction d'un capital symbolique, marqué par des compétences techniques et d'un capital social. La reconversion de $\mathrm{M}$. Pascal en entrepreneur de l'éducation s'appuie effectivement sur un capital symbolique issu de son parcours scolaire, de son expérience de l'éducation acquise au cours de sa fonction à l'INA. Néanmoins, le contexte actuel de "l'aide décentralisée » (Bierschenk, Chauveau et Olivier de Sardan, 2000) modifie quelque peu la physionomie du capital social: les relations acquises au sein des structures qui ouvrent à des ressources financières exigent une obligation de résultats, d'abord, à un niveau local ou communautaire dans la phase expérimentale, ensuite à un niveau national ou à échelles élargies. L'aide décentralisée présuppose ainsi des retombées collectives ou publiques que n'impliquait pas le système précédent.

7 Ces reconversions de fonctionnaires, comme celle de M. Pascal, conduisent à interroger la signification du fonctionnariat au Burkina Faso. Déjà dans les années 1960, la position de fonctionnaire était perçue comme une opportunité pour accroitre ses relations et son capital symbolique(Skinner, 1974: 85). C'est ce que confirment plus tard les analyses de Labazée où les compétences techniques, la reconnaissance symbolique qui leur est liée et les relations sociales acquises en tant que fonctionnaires permettent des reconversions dans des domaines variés tels que l'action culturelle ou politique et les sphères économiques du commerce ou du management. Le contexte socio-politique et économique actuel de désengagement de l'État et de l'aide décentralisée contribue à renforcer ces représentations du fonctionnariat comme une position. Néanmoins, au sein de la conjoncture actuelle, les bénéfices personnels ne suffisent pas. Les bailleurs de fonds incitent les entrepreneurs à composer avec une pluralité d'acteurs et de référentiels. 


\section{L'éducation bilingue : une composition polymorphe}

8 La réorientation de la trajectoire de $\mathrm{M}$. Pascal prend forme à un carrefour de différents facteurs et grâce à de multiples acteurs. En cela, l'éducation bilingue est une composition polymorphe qui résulte « du sens du jeu» (Bourdieu, 1994) du promoteur. C'est de sa capacité à justifier de manière pluraliste son entreprise, de son habile usage de différents référentiels de sens et de multiples normes que dépend la réussite de son initiative. Trois grands régimes de justifications sont mobilisés par le promoteur de l'éducation bilingue ${ }^{8}$ : d'abord, l'entreprise bilingue est présentée comme un produit rentable et économique sur un marché éducatif concurrentiel (justification économique ou marchande); deuxièmement, un ensemble d'arguments sont orientés vers la participation active des populations locales à leur développement, ainsi qu'au développement du pays (justification communautaire ou civique). Enfin, le régime de justifications qui pourrait être qualifié de culturaliste et identitaire, au sein duquel l'éducation bilingue est présentée comme assurant la perpétuité des traditions et valorisant les langues et les cultures locales, est étroitement liée avec une posture « inspirée » (Boltanski et Thévenot, 1991) et désintéressée du promoteur. En outre, audelà de la rhétorique, M. Pascal mobilise quatre types d'acteurs incontournables au succès de son entreprise : l'ONG-partenaire, le niveau étatique, les populations locales, le personnel enseignant.

\section{Justification marchande : l'éducation bilingue, un produit éducatif rentable}

9 L'éducation bilingue est une innovation pédagogique dont la phase expérimentale s'est déroulée de 1994 à 1998 au sein de deux écoles pilotes avec 55 enfants âgés de 9 à 14 ans. Elle repose sur une méthode d'apprentissage de la langue française élaborée par M. Pascal et une équipe de linguistes et de pédagogues. Cette alternative pédagogique pour l'enseignement de base s'appuie sur les langues locales qui sont intégrées au sein d'un bilinguisme "additif » langue locale ${ }^{9}$ - français. De par cette méthode, l'éducation bilingue est présentée comme un produit efficace et rentable dans un contexte éducatif marqué par la rareté des ressources: elle permettrait selon $\mathrm{M}$. Pascal de réduire l'enseignement de base d'une année. En effet, selon lui et ses collaborateurs, au terme de cinq années - et non de six comme dans le système d'enseignement « classique $~^{10}{ }^{10}$-, les élèves pourraient présenter leur examen pour l'obtention du certificat d'études de base et les résultats seraient plus satisfaisants que dans l'enseignement de base classique. ${ }^{11}$.

10 Cette première justification répond aux injonctions internationales de rentabilité des investissements en éducation dans une conjoncture caractérisée par l'importance des décisions des bailleurs de fonds face aux difficultés de l'État dans ses responsabilités éducatives. Ce contexte particulier impose aux initiatives privées d'être rentables et efficaces par rapport au taux de scolarisation du pays. C'est pour cette raison que la justification économique est devenue le premier argument du promoteur pour l'extension de son entreprise. L'ONG-partenaire a d'ailleurs renouvelé son soutien en finançant l'extension géographique à d'autres provinces et linguistique à d'autres langues. De plus cet argument semble avoir convaincu le ministère de l'enseignement 
de base qui s'est intéressé à ces expériences et les a reconnues semi-officiellement. En effet, le 8 juin 2002, le ministre de l'enseignement de base et de l'alphabétisation de l'époque, Mathieu R. Ouédraogo, a adressé une circulaire sur la transformation des écoles classiques en écoles bilingues aux directions régionales de l'enseignement de base et de l'alphabétisation afin d'accorder à toute communauté qui le souhaite la transformation de son école en une école bilingue avec l'autorisation du ministère. Enfin, il a également convaincu le réseau privé par cet argument : l'enseignement catholique transforme progressivement ses écoles primaires en écoles bilingues et trois écoles privées non confessionnelles bilingues viennent de voir le jour en raison de la rentabilité de ce « produit éducatif ».

\section{Justification communautaire : une auto-éducation}

11 La justification économique est étroitement articulée à l'existence d'un argumentaire "communautaire». Cette seconde justification épouse en partie la rhétorique participative promue par les organisations internationales. Les nouvelles modalités de l'aide décentralisée ont tendance à se ponctuer au départ de franges intermédiaires situées à un niveau local dont elles attendent les bons résultats pour maintenir et/ou étendre leur soutien. Les organisations internationales attendent un degré élevé de participation des populations locales (Bierschenk, 2000).

12 L'investissement dans le terroir signe le début de la reconversion de M. Pascal. Celui-ci, après de nombreuses rencontres avec les groupements villageois de sa région les incite à se constituer en association, c'est-à-dire, en un collectif communautaire crédible aux yeux des bailleurs de fonds et nécessaire pour «les modalités décentralisées de l'aide actuelle ». L'association Manegdbzãnga, dont le nom en moore qui signifie "développement pour tous " renvoie directement à la rhétorique participative, voitle jour en 1991 à Nomgana avec 26 groupements villageois et l'appui technique et financier d'une ONG suisse importante. L'investissement constant des membres de l'association, qui considèrent aujourd'hui Nomgana et ses environs comme le berceau et le bastion de l'éducation bilingue, a joué un rôle important dans l'extension de l'entreprise. Cet ancrage local et communautaire constitue l'un des principaux arguments de la rhétorique de M. Pascal qui a su emporter l'adhésion des bailleurs de fonds.

13 Au Burkina Faso, dans le cadre de l'éducation de base, la prégnance de la rhétorique participative n'est pas un fait neuf. La formule pédagogique et l'idéologie de l'éducation bilingue répondent à cet impératif participatif en renouant avec une formule éducative apparue dès les premières années de l'indépendance. En Haute-Volta, les effets de la " critique identitaire ${ }^{12}$. de l'institution scolaire ont été minimes, comparativement à la « critique ruralisante » de l'école (Bianchini, 2004). Celle-ci influencera les premières grandes réformes scolaires recourant à l'introduction des langues et des cultures locales. Elles ont jeté les bases d'une relation étroite entre langues et cultures locales, institution scolaire et développement socio-économique du pays (André, 2007). Cette contestation ruralisante à l'égard de l'école est le fait d'experts français (Bianchini, 2004), les premiers "développeurs", qui proclament la nécessité de réformer l'école voltaïque en vue d'enrayer le sous-développement et d'engendrer la rentabilité d'une institution scolaire encore trop élitiste et éloignée des communautés rurales. L'exigence d'une éducation plus proche des ruraux par le biais des langues et des 
cultures locales, ainsi qu'une formation technique aux activités de production (culture, maraîchage), visaient leur encadrement et leur participation active à leur éducation et à leur développement ainsi qu'au redressement socio-économique du pays. De cette critique, naîtra progressivement l'éducation informelle essentiellement située en zone rurale. Les réformes éducatives de Sangoulé Lamizana ${ }^{13}$ et de Thomas Sankara (SNGCDR : 1986), en lien étroit avec leurs auto-ajustements respectifs. ${ }^{14}$, chercheront à développer cette formule pour l'éducation formelle au niveau primaire en intégrant les langues et les cultures locales afin de favoriser l'investissement des parents dans l'éducation de leurs enfants. Un des objectif était d'établir un lien étroit entre l'éducation et la production par le biais d'activités de production telles que le maraîchage, le tissage, etc., afin d'élaborer une possibilité de prise en charge des coûts de l'éducation par les populations locales, bref une auto-éducation et un autodéveloppement. Reposant sur une idéologie de promotion communautaire, ces réformes auraient essuyé des échecs (Sanou, 2003a) notamment parce qu'elles allaient à l'encontre des représentations des populations locales qui percevaient l'institution scolaire franco-française comme la voie du succès et des promotions individuelles.

En s'appuyant sur les expériences d'introduction des langues et des cultures locales qui l'ont précédée, l'entreprise bilingue cherche à satisfaire la rhétorique de décentralisation encourageant la responsabilité des populations locales dans leur processus d'éducation et de développement. M. Pascal et ses collaborateurs présentent cette innovation comme permettant de rapprocher les familles de l'école, et ainsi, de les responsabiliser par rapport à l'éducation de la collectivité. En outre, les activités de production visent à contribuer aux revenus des ménages afin qu'en retour ils assument une part des coûts occasionnés par la scolarité de leurs enfants. Enfin, les différentes activités de production participent à l'ancrage communautaire : en effet, les activités d'élevage, de maraîchage, de tissage visent à rompre le lien de continuité imaginaire entre institution scolaire et fonction publique, qui aujourd'hui est saturée, et à lutter contre l'exode rural. Les promoteurs de l'éducation bilingue présentent leur initiative comme " une école productive ", " favorable au développement local et celui du pays ", en "améliorant de manière significative l'efficacité externe de l'école en ancrant les écoles bilingues dans leur contexte de développement local afin que les sortants de ces écoles soient utiles à eux-mêmes en tant qu'acteurs et bénéficiaires de développement au lieu d'être des candidats à l'exode, à la paresse et à la délinquance " (Ilboudo Taryam, $2004: 4)$. Cette justification, qui souligne l'intérêt des activités de production, rencontre les attentes d'une partie des populations locales dont les imaginaires situent progressivement de nouvelles trajectoires de la réussite en dehors de la fonction publique (Banégas et Warnier, 2001).

\section{Le régime de justifications culturalistes}

D'autres discours de justification, qui complètent les deux précédents, s'appuient sur un registre culturaliste de promotion de la culture «burkinabé » et particulièrement de ses langues nationales. Ainsi, la promotion de la culture burkinabé passe par l'usage de manuels spécifiques à l'école bilingue qui ont été élaborés par une équipe de linguistes et de pédagogue, parmi lesquels M. Pascal. Ces manuels reposent sur des méthodes pédagogiques dites traditionnelles telles que les séances de causerie précédant les séances de lecture, mais aussi sur une utilisation sélective de certaines valeurs jugées 
positives telles que « le respect des aînés », « la solidarité rurale » et « le dynamisme de la vie collective traditionnelle », "l'amour du travail communautaire » qui sont en fait des stéréotypes qui surévaluent « les fonctions intégratrices et communautaires » des zones rurales (Olivier de Sardan, 1995). Dans le même esprit, la valorisation de la culture et des traditions burkinabè est véhiculée à travers des contes, des chants et des danses, ainsi que les activités dites de production. Ainsi la formation pratique au cours de laquelle les enfants sont initiés aux activités du milieu dans un but communautaire s'appuie-t-elle également sur des supposées valeurs culturelles et identitaires.

Ces valeurs et ces activités dites "traditionnelles» et "culturelles » découlent de l'appareil développementaliste qui repose en matière de culture sur le mythe de la communauté consensuelle. A ces méthodes se conjuguent l'apprentissage du français et des formations aux principes chers aux organismes financiers tels que «la citoyenneté », "l'équité entre les genres». Cet assemblage permet d'emporter l'adhésion d'acteurs décisifs tels que les bailleurs de fonds et de développer les initiatives d'écoles bilingues. La dimension culturelle est pour certains partenaires techniques et financiers un impératif: d'une part, dans la mouvance onusienne humaniste, elle répond au respect de chaque être humain d'être scolarisé dans sa langue et sa culture et sert la diversité culturelle et linguistique. D'autre part, cette dimension permet de mieux faire accepter l'aspect économique du projet de développement (Sanchez-Arnau et Desjeux, 1983 : 37). Quoiqu'il en soit, l'intégration de cette norme a consacré $\mathrm{M}$. Pascal comme le leader d'une formule pédagogique promouvant la culture burkinabè au point qu'il reçoive le prix de la personnalité francophone pour sa contribution à la diversité culturelle et au multilinguisme. La justification culturelle de son entreprise a contribué à l'accumulation d'un capital symbolique personnel et la dote de puissantes valeurs morales. Celles-ci apparaissent dans ses discours, en particulier ceux qu'il adresse aux enseignants dont certains lui vouent une admiration profonde :

Du courage, parce que notre combat ne fait que commencer! Beaucoup croient que nos langues ne véhiculent pas de connaissance, et que nos cultures n'ont pas lieu d'être à l'école. Pourtant l'introduction de nos langues et de nos cultures conduit à de très bons résultats au CEP. Nous pouvons presque crier victoire, nous marchons dans la bonne direction. C'est un défi que vous lancez ! Vous les aînés qui allumez le flambeau de la victoire et qui ouvrez les chemins aux autres qui feront encore mieux! Vous devez être à la hauteur, et même au-delà, au niveau de tous les concours. Au-delà des résultats académiques, au concours culturel de la francophonie, sur huit écoles gagnantes, 6 écoles étaient des écoles bilingues. [...] Du courage, pour que croient tous ces incrédules en la force de nos langues et de nos cultures! Il faut que nos fils s'expriment, chantent et dansent dans leurs langues et leurs traditions! (M. Pascal, août 2004, Bobo-Dioulasso).

17 L'efficacité de la justification culturaliste indique que le champ de la culture est un « monde économique inversé » "reposant sur la censure de l'intérêt économique » et " assurant des profits spécifiques de désintéressement à ceux qui se plient à ses règles, crée les conditions d'un véritable intérêt au désintéressement» (Bourdieu, 1994 : 200-209). Par le recours et la mise en avant du registre culturaliste, M. Pascal paraît en quelque sorte, lui-même, suffisamment désintéressé pour donner à son travail les allures d'un combat au seul service d'une éthique et de convictions personnelles. 


\section{L'école du développement : de la rhétorique aux pratiques effectives}

\section{Les revendications francophiles}

18 Si la justification culturaliste est centrale pour l'obtention du soutien des bailleurs de fonds, paradoxalement, l'entreprise bilingue ne trouve pas ses assises dans les revendications identitaires de populations en faveur de leurs langues et de leurs cultures, mais bien dans les revendications « francophiles » de groupements paysans, désireux d'acquérir un capital linguistique « légitime ", à savoir le français.

L'association Manegbzanga, mise en place par le promoteur, a fait de «son cheval de bataille l'alphabétisation et la formation». Lorsque $50 \%$ de ses membres ont été alphabétisés en moore, ceux-ci ont voulu apprendre le français, afin, comme ils l'expliquent, «d'aller en ville » et "d'accueillir les visiteurs du développement », soit d'accéder à des sphères qui leur permettent de développer plus facilement leur village. M. Pascal a répondu à ces demandes en mettant au point, sous la coupe de l'ONG-Suisse et avec une série d'autres linguistes et pédagogues, la méthode d'apprentissage de la langue française à partir des acquis de l'alphabétisation en langue nationale. Celle-ci a permis aux villageois d'apprendre rapidement le français. Ayant bénéficié de cette double alphabétisation et souhaitant les mêmes avantages pour leurs enfants non scolarisés, ils ont sollicité l'aide du promoteur. Ils souhaitaient que les enfants de la tranche d'âge trop élevée pour bénéficier de l'éducation formelle et trop jeune pour les centres d'alphabétisation en langue profitent, eux aussi, de cette méthode.

L'histoire de l'institution scolaire francophone, placée stratégiquement par les administrateurs coloniaux au centre de la rentabilité et de l'efficacité de leur entreprise, par la formation in situ d'auxiliaires indigènes compétents, promis de surcroît à une ascension sociale fulgurante. ${ }^{15}$, a consacré dans les imaginaires collectifs la langue française et l'école ${ }^{16}$. comme vecteurs de réussite. Néanmoins, la conjoncture actuelle en matière de débouchés au sein de la fonction publique sur la base d'une scolarisation francophone ne correspond plus à cette situation d'antan de convertibilité immédiate du "capital scolaire francophone»en d'autres types de "capitaux", économique et surtout politique (Bianchini, 2004). Celle-ci est en effet saturée et les populations en ont progressivement conscience (Banégas et Warnier, 2001). Néanmoins, l'histoire de l'entreprise bilingue montre que la langue et la culture françaises restent des passerelles vers la promotion sociale, notamment par la maîtrise des instruments de développement. La connaissance de la langue française est perçue par les groupements villageois de la région de Nomgana comme une condition nécessaire pour capter les ressources du développement. Le nombre de partenaires techniques et financiers dont bénéficie l'association n'est en ce sens pas anodin. La primauté des activités de formation et d'alphabétisation bilingue au sein de l'association et leur visée d'acquisition rapide de la langue française témoignent de l'importance que peut revêtir la «demande » d'un projet de développement pour des paysans analphabètes (Laurent, 1998).

21 Le registre culturaliste décrit plus haut ne correspond donc pas aux intentions prioritaires des populations locales qui veulent apprendre le français. L'engouement progressif des populations révèle leur réappropriation de l'éducation bilingue comme 
«l'éducation du développement»: dans les représentations locales, l'éducation bilingue offre d'abord une scolarité plus fiable parce que placée sous la coupe d'une structure de développement. Dans les représentations des parents, ces écoles sont susceptibles de produire de véritables " courtiers en développement ", des médiateurs capables d'être à l'aise dans des univers culturels multiples par la maitrise de différents codes, normes et langues.

\section{L'éthique culturaliste et la vocation d'enseignants désillusionnés}

En dépit des parents qui s'approprient l'éducation bilingue comme l'école du développement, le personnel-enseignant est particulièrement sensible au registre culturaliste. Initiés à la méthodologie décrite plus haut et à l'idéologie culturaliste de l'éducation bilingue, les enseignants sont acquis à la nécessité de promouvoir leurs langues et leurs cultures. La fonction d'enseignant bilingue est vécue d'une certaine manière comme une vocation de soutien des langues, des cultures et des traditions nationales. Ils se sentent investis d'une mission telle qu'elle leur permet dans le même temps de redécouvrir leurs langues et leurs cultures à travers l'expérimentation. Conduits à quitter leur village pour mener leurs études secondaires et supérieures, ces intellectuels qui ont voyagé et fréquenté la ville, entretiennent parfois un sentiment de déracinement. Les affectations en zones rurales ne sont pas toujours aisées puisqu'ils ne possèdent plus l'entière maîtrise des codes culturels qui les régissent. Leur apportant certains éléments culturels de ce qu'ils pensent avoir perdu, l'enseignement bilingue représente pour eux une possibilité de se réimplanter dans une sphère locale. Placés au centre de l'expérimentation par l'ONG-partenaire comme de véritables médiateurs, ils participent à l'invention d'une certaine tradition et à sa diffusion.

En même temps, les enseignants ne sont pas dupes des réalités villageoises et leur investissement au sein de l'entreprise bilingue en faveur des langues et cultures nationales connaît d'autres raisons. La pérennité des financements de l'éducation bilingue implique l'exigence capitaliste de la rentabilité de l'entreprise, à savoir une scolarisation efficace à moindre coût et les bons résultats sont fortement tributaires du travail fourni par le personnel-enseignant. Exception faite de certaines pratiques clientélistes qui touchent l'ensemble des fonctions dans l'éducation bilingue, le recrutement des enseignants s'effectue moins sur la base de leurs qualifications obtenues dans les ENEP (Ecoles Nationales des Enseignements primaires) que sur leur motivation à l'égard de la « cause identitaire et culturelle » de l'enseignement bilingue. Généralement, les enseignants motivés se voient proposer d'intégrer le système. Nos enquêtes ont montré que l'entrée dans l'éducation bilingue des enseignants est souvent en étroite relation avec la figure inspirée de M. Pascal. Pour plusieurs enseignants, la proposition de rejoindre l'éducation bilingue s'est faite par « le parrain » lui-même :

J'avoue que, au temps où j'étais à l'ENEP, on ne connaissait pas le bilingue, mais j'ai eu la chance de pouvoir approcher le parrain et de connaître par lui-même le bilingue. En fait, au début, je n'étais pas sûr, mais le parrain m'a beaucoup encouragé. J'ai beaucoup d'admiration pour le parrain et son combat pour nos langues! (Enseignant peul moorephone, juillet 2004, Nomgana).

Si les enseignants sont, à l'époque coloniale, bénéficiaires d'une forte promotion sociale et porteurs d'une certaine aura auprès des populations.$^{17}$, au Burkina Faso, ils subissent par la suite une dévaluation économique et symbolique vertigineuse de leurs fonctions. Leurs salaires et leurs formations ont été au cours des différents plans d'ajustements 
structurels et des différentes réformes éducatives fortement entaillés. En outre, la croissance de la privatisation participe également à la dévaluation de leurs fonctions. Engagés sans devoir être passé dans une ENEP. ${ }^{18}$, souvent sous-payés, certaines écoles privées participent à la « désintellectualisation » de la figure de l'instituteur. De plus, l'ajustement éducatif a eu pour conséquence une réduction de leur formation d'une année au lieu de deux au sein des ENEP. Les enseignants ne considèrent pas leur fonction comme une vocation, mais bien comme un moyen de survivre.

Au Burkina Faso, toute innovation éducative parce qu'elle implique un investissement plus important de la part de son personnel attribue aux enseignants une prime mensuelle de 15.000 CFA. Dans le cas de l'entreprise bilingue, les enseignants reçoivent également une compensation financière par jour de formation. Néanmoins, bien que cet aspect puisse être un attrait pour l'inscription dans l'entreprise bilingue, il ne compense pas, à leurs yeux, leur investissement personnel intense ni l'importance de leurs responsabilités. Les enseignants bilingues ${ }^{19}$. sont pourtant, au moins durant leurs premières années dans ces écoles, engagés dans leur mission, réalisant leur travail comme s'il s'agissait d'un devoir moral, ou plutôt d'une vocation. Il semble que ce soit davantage par l'attachement à une éthique qu'à un supplément financier que les enseignants vivent leur fonction dans l'entreprise bilingue comme une vocation ${ }^{20}$.

M. Pascal, le leader de l'entreprise bilingue, investit ses employés d'une mission de sauvegarde et de promotion des langues et des cultures locales. Les nombreuses formations auxquelles doivent participer les enseignants durant les congés scolaires participent à la constitution d'une éthique culturaliste qui les charge de responsabilités et d'obligations morales à l'égard de leurs langues, de leurs cultures et de leurs communautés, même si au départ certains enseignants se sont inscrits dans le bilingue pour des questions matérielles. C'est surtout à cause d'enjeux symboliques qu'ils se responsabilisent à l'égard de leur fonction. En effet, l'expérience de l'enseignement bilingue et les formations qu'ils suivent régulièrement participent de leur implication dans la valorisation de leur culture et de leur langue maternelle. Ainsi naît souvent une vocation pour l'enseignement bilingue qui stimule leur entrain au travail. La participation à une expérience financée par une ONG contribue aussi à l'ancrage de la certitude que l'enjeu est de taille. On trouve ainsi de nombreux enseignants qui cumulent dans leurs trajectoires, les expériences éducatives sous la coupe de bailleurs de fonds. Outre l'appointement financier supplémentaire qu'elles fournissent, la recherche de responsabilités sous des autorités fiables, ainsi que des missions contribuant à une certaine "réintellectualisation » de l'image du corps enseignant les stimulent.

\section{Conclusion}

27 Sous les incitations des bailleurs de fonds et suite au désengagement de l'État, le champ éducatif burkinabè se reconfigure et stimule des initiatives privées telles que l'éducation bilingue. Le champ éducatif et ses principaux décideurs actuels, les organisations internationales, imposent aux dynamiques entrepreunariales de satisfaire à différentes exigences: une efficacité économique dans un contexte de rareté des ressources, un ancrage communautaire et des retombées collectives, une cause «noble» dans le sens où sa principale visée n'est pas le profit personnel. La 
réussite de la reconversion professionnelle de $\mathrm{M}$. Pascal s'explique par sa capacité à jongler avec les différents référentiels chers aux bailleurs de fonds : les rhétoriques économiste, participative et communautaire, culturaliste.

Parce que la conjoncture éducative se caractérise par une redistribution des responsabilités où les populations locales sont invitées à jouer un rôle prépondérant, le succès de toute entreprise nécessite de tenir compte de leurs aspirations propres. Le succès de l'éducation bilingue s'explique ainsi en ce que cette initiative répond aux attentes des populations locales d'être connectées au monde du développement. A cela s'ajoute le fait que les représentations et les attentes des populations locales à l'égard de l'école et de l'Etat évoluent: l'entrain de certaines communautés pour l'éducation bilingue exprime une remise en question de la scolarité classique francophone sous la coupe étatique. Les responsables de ces " écoles du développement ", semblent susciter plus de crédit. Quant à leurs formules éducatives, elles font figure de voies plus sûres de promotion individuelle dans un contexte où la fonction publique comme débouché classique de l'école primaire est affecté par la situation socio-économique. La réussite de M. Pascal tient aussi à ce qu'elle revalorise la fonction enseignante, tout autant par sa réintellectualisation que par les avantages matériels.

\section{BIBLIOGRAPHIE}

André, Géraldine, 2007, «Ecole, langues, cultures et développement. Une analyse des politiques éducatives, linguistiques et culturelles », Cahiers d'études africaines, 186.

Banegas, Richard et Warnier, Jean-Pierre, 2001, « Nouvelles figures de la réussite et du pouvoir », in Politique africaine, $82: 5-23$.

Bianchini, Pascal, 2004, Ecole et politique en Afrique noire. Sociologie des crises et des réformes du système d'enseignement au Sénégal et au Burkina Faso (1960-2000), Paris, Karthala.

Bierschenk, Thomas, Chauveau, Jean-Pierre et Olivier De Sardan, Jean-Pierre, 2000, Courtiers en développement. Les villages africains en quête de projets, Paris, APAD-Karthala.

Boltanski, Luc et Thevenot, Laurent, 1991, De la justification. Les économies de la grandeur, Paris, Gallimard.

Bourdieu, Pierre, 1994, Raisons pratiques. Sur la théorie de l'action, Paris, Seuil.

Ilboudo Taryam, Paul, 2004, L'école Bilingue. Une alternative au système éducatif de base formel au Burkina, Ouagadougou : MEBA OSEO.

Jezequel, Jean-Hervé, 2005, « Les enseignants comme élite politique en AOF (1930-1945). Des meneurs galopins dans l'arène politique », Cahiers d'Études africaines, XLV (2), 178 : 519-543.

Lange, Marie-France, 2003, «Ecole et mondialisation. Vers un nouvel ordre scolaire ? ", in Cahiers d'études africaines, XLIII (1-2), 169-170 : 143-166.

Laurent, Pierre-Joseph, 1998, Une association de développement en pays mossi. Le don comme ruse, Paris, Karthala. 
Labazee, Pascal, 1988, Entreprises et entrepreneurs du Burkina Faso. Vers une lecture anthropologique de l'entreprise africaine, Paris, Karthala.

Meba, 2004, Plan décennal de développement de l'éducation de base 2000-2009, Ouagadougou.

Ministere de l'Education Nationale et de la Culture, 1976, Réforme de l'éducation. Dossier initial, Ouagadougou,

Olivier De Sardan, Jean-Pierre, 1995, Anthropologie et développement. Essai en sociologie du changement social, Paris, Apad/Karthala.

Pilon, Marc, 2002, « L'évolution du champ scolaire au Burkina Faso : entre diversification et privatisation ", ARES, $3: 143-165$.

Sanchez Arnau, Juan Carlos et Desjeux, Dominique, 1994, La culture clé du développement, Paris : L'Harmattan.

Sanou, Fernand, 2003a, «Colonialisme, éducation et langues : hier et aujourd'hui », in Burkina Faso. Cent ans d'histoire, 1895-1995 (Actes du premier colloque international sur l'histoire du Burkina Ouagadougou, 12-17 décembre), Paris, Karthala, Tome 2 : 1791-1817.

SGN-CDR, 1986, L'école révolutionnaire burkinabé, Ouagadougou.

Skinner, Elliot, 1974, African Urban Life. The transformation of Ouagadougou, Princeton, University Press.

Tidjani Alou, Mahaman, 2001, « Globalisation : l'État africain en question », Afrique contemporaine, numéro spécial : 11-23.

Weber, Max, 1964, L'éthique protestante et l'esprit du capitalisme, Paris, Plon.

Zagre, Pascal, 1994, Les politiques économiques du Burkina Faso, Paris, Karthala.

\section{NOTES}

1. On qualifie la trajectoire de l'entrepreneur étudié « d'exemplaire » non pas pour le succès de l'entreprise qui est sans cesse affirmé et affiché par les représentants de l'initiative, mais parce qu'elle permet de saisir certains traits typiques de l'entrepreunariat africain dans le champ éducatif. Ainsi, celui que nous appellerons dans le cadre de ce texte M. Pascal est devenu le représentant au Burkina Faso de l'ONG qui a soutenu à la base son initiative éducative. Parti d'un centre d'alphabétisation pour adultes en 1986, il est responsable en 2005 de 110 écoles primaires parmi lesquelles 75 sont publiques, 32 privées catholiques et 3 privées non confessionnelles.

2. Ce texte repose sur les résultats d'une enquête socio-anthropologique dont le point de départ empirique est l'éducation bilingue, une alternative pédagogique expérimentée au niveau de l'enseignement fondamental au Burkina Faso. L'enquête s'est déroulée du mois de févier 2004 au mois de septembre 2004, à la fois en zone urbaine, auprès des acteurs impliqués dans une école bilingue à Koudougou (parents, enseignants, enfants etc.), d'autre part, en zone rurale, à Nomgana, berceau de l'expérience. Toutes les méthodes classiques de l'anthropologie ont été mobilisées: observation participante, discussions et échanges informels individuels et en groupe. Une observation participante a aussi été conduite dans trois centres de formation à la méthode bilingue adressée à des enseignants: Nomgana, Bobo-Dioulasso et Fada'N Gourma. Des entretiens plus structurés ont été réalisés avec quarante enseignants, trente parents ou responsables d'enfants. Les responsables de l'éducation bilingue ont été rencontrés plusieurs fois dans le cadre d'évènements et de manifestations liés à l'entreprise et un long entretien a été réalisé avec $\mathrm{M}$. Pascal. 
3. Plan décennal de développement de l'éducation de base 2000-2009, MEBA, 1999.

4. Aujourd'hui, l'INA est devenu DRINA, la direction de la recherche des innovations en éducation non formelle et en alphabétisation et dont certaines de ses missions sont la valorisation des langues et des cultures locales et l'alphabétisation.

5. L'éducation au Burkina Faso se partage entre l'éducation formelle aux enfants en français et l'éducation informelle généralement en langues nationales adressée aux jeunes gens et aux adultes.

6. Selon les termes employés lors d'un entretien en juillet 2004 par le président de l'association, Manegdbzãnga, qui chapeaute l'éducation bilingue.

7. Selon les termes employés par le président de l'association, Manegdbzãnga.

8. D'une certaine manière, bien qu'ils soient élaborés dans un contexte largement différent, ces régimes de justifications correspondent respectivement à la cité marchande, la cité civique, enfin, la cité inspirée telles que développé dans l'ouvrage de Boltanski et Thévenot (Boltanski et Thévenot, 1991).

9. Celle-ci n'est pas nécessairement la langue maternelle de l'enfant, c'est la langue de la localité. Jusqu'à présent, 7 langues nationales ont été introduites comme médiums d'enseignement aux côtés du français : moore, dioula, fulufulde, dagara, lyeele, gulmancema, bisa.

10. Cette qualification de l'enseignement non bilingue devenue courante tant du côté « des non bilingues" que des «bilingues" n'est pas anodine. Elle révèle la normalisation historique francophone de l'institution scolaire présente dans les imaginaires.

11. Selon les sources des écoles bilingues, «les écoles bilingues qui ont pu passer avec une autorisation spéciale du Ministère de l'Enseignement de base les examens officiels du certificat d'études primaires ont obtenu en 4 ans un taux de succès de $52,83 \%$ alors que la moyenne nationale était de 42\% » (Ilboudo Taryam, 2004 : 5).

12. La critique identitaire promeut la culture, les traditions et l'identité noires et a été portée par les mouvements de la négritude «francophone " dont Léopold Sédar Senghor est le chef de file, ou « afrancophone » de Cheikh Anta Diop. Selon Bianchini, elle a influencé un grand nombre de réformes scolaires dans les années 1970 en Afrique francophone (Bianchini, 2004).

13. 1976, Réforme de l'éducation. Dossier initial, Ouagadougou : Ministère de l'éducation nationale et de la culture.

14. Le «Plan d'ajustement structurel avant la lettre, la garangose (1966-1975)» dont l'un des moyens du redressement des finances publiques est une "politique agricole» et «un encadrement du monde rural » aura des effets non négligeables, qui se prolongeront jusqu'à la veille de la révolution, (Zagre, 1994), sur la tournure des politiques éducatives. Quant à Thomas Sankara, sa méfiance à l'égard des institutions de Bretton Woods et l'idéologie autonomiste du CNR conduisent à un " auto-ajustement » visant à composer avec ses propres forces, à établir des dépenses conformes aux ressources du pays (Zagre, 1994). Ce «P.A.S. maison » aura de sérieuses implications sur l'éducation, notamment le renforcement de ses liens avec le développement et les langues nationales.

15. Pour une illustration littéraire de ce phénomène, voir le livre du célèbre Hampaté Ba, (1992), L'étrange destin de Wangrin ou Les Roueries d'un interprète africain, Paris : Editions 10-18.

16. L'éducation au Burkina Faso se partage entre les structures formelles de l'enseignement (l'école primaire et secondaire en français issues de la colonisation adressée aux enfants et aux jeunes respectant les limitations d'âge) et informelles (les centres d'éducation et de formation à destination des adultes et des jeunes qui n'ont pas été scolarisés

17. Jean-Hervé Jézéquel explique que pendant la colonisation, les instituteurs apparaissaient aux Européens « de détribalisés et de semi-intellectuels, suspectés d'être des contestataires potentiels 
de l'ordre colonial. Les sociétés africaines, pour leur part, associent la figure de l'instituteur à un ensemble de représentations ambiguës. S'il peut être admiré pour son paraitre et son savoir, il est aussi considéré comme un agent de l'oppression coloniale [...]. » (Jézéquel, op. cit., p. 528).

18. Ecole nationale des enseignements primaires.

19. - Le cadre de cet article ne nous permet pas de distinguer les enseignants selon leurs trajectoires, ce qui constitue l'objet d'un autre travail. Les variations au sein de leurs trajectoires ne permettent pas de parler d'un groupe socio-professionnel homogène, quoiqu'ils partagent une matrice représentationnelle commune de la fonction enseignante, surtout dans la jeune génération, et de leur inscription au sein de l'enseignement bilingue.

20. Weber (1964) a montré que le capitalisme s'est développé entre autres par une conception du travail « en tant que fin en soi, vocation (Beruf) », « comme une obligation morale ».

\section{RÉSUMÉS}

Cet article est consacré à l'analyse de la genèse et de l'expansion, au Burkina Faso, d'une expérimentation pédagogique, celle de l'éducation bilingue, placée sous la coupe d'une ONG. À travers la trajectoire du succès de cette entreprise dans le champ de l'enseignement de base, ce texte tente de dépasser les singularités de l'étude de cas. Il vise à apporter des éléments de réponse quant au type de compétences que requièrent les entrepreneurs africains pour faire aboutir leurs démarches dans un contexte éducatif caractérisé par une forte privatisation et une importance de l'aide décentralisée.

This article analyse the genesis and the expansion, in Burkina Faso, of a teaching experimentation, the bilingual education, directed by an ONG. Through the trajectory of the success of this enterprise of basic teaching, this text tries to exceed the singularities of the case study. It aims at bringing brief replies about the type of competences which require the African entrepreneur to make succeed their steps in an educational context characterized by a strong privatization and an importance of the decentralized assistance.

\section{AUTEUR}

\section{GÉRALDINE ANDRÉ}

Anthropologue, FUCAM/GRESAS (Facultés universitaires catholiques de Mons/Groupe de recherche sociologie action sens). 